\title{
Estudio epidemiológico en el Área de Salud de Entre Ríos
}

\author{
Víctor Manuel Sánchez Castilloa
}

a Centro de Salud Municipal de Entre Ríos, Vila Entre Ríos, Municipio Caroebe, RR. Brasil.

Correspondencia: Víctor Manuel Sánchez Castillo, Rua Cecília Brasil ${ }^{\circ} 317$, Apto 04, Centro, Boa Vista, RR. Brasil. CEP: 69.301-080. Telf.: 559588042531 ,

correo electrónico: cubanbr2010@hotmail.com

Recibido el 12 de noviembre de 2010.

Aceptado para su publicación el 4 de enero de 2011.

\section{RESUMEN}

Objetivo. Conocer las principales enfermedades y problemas de salud en la comunidad rural de Entre Ríos.

Diseño. Estudio descriptivo transversal.

Emplazamiento. Atención Primaria. Área de salud de Entre Ríos, Caroebe, RR. Brasil. Mediciones principales. Se registraron datos socio demográficos y de morbilidad de pacientes con diagnóstico de enfermedades crónicas, consultas de ambulatorio y de urgencia y factores de riesgo.

Resultados. Predominaron los casos dentro del grupo etario de 0-10 años $(36,09 \%)$ y del sexo femenino $(61,4 \%)$. Los pacientes con enfermedades crónicas identificados en el Programa de Hipertensión y Diabetes Mellitus constituyeron el 2,98\% (72 casos) del universo de estudio. Las enfermedades tropicales malaria, dengue y leishmaniosis son frecuentes con 16 casos $(0,66 \%)$, 20 casos $(0,81 \%)$, y 24 casos $(0,99 \%)$ respectivamente. Las enfermedades respiratorias e intestinales representan el $13,26 \%$ y $10,36 \%$ respectivamente. Se identifica el predominio de adolescentes embarazadas (15 - 18 años) con el 41, $66 \%$ de los casos atendidos en el Programa de Prenatal.

Conclusiones. Los pacientes clasificados con enfermedades crónicas no transmisibles reciben una atención controlada y sistemática. Predominan los casos de pacientes atendidos por enfermedades transmisibles, encontrándose fundamentalmente las infecciones respiratorias e intestinales agudas. Las enfermedades tropicales como paludismo, dengue y leishmaniosis constituyen problemas de salud típicos en esta región. Del total de pacientes, el 63,28\% recibió atención de urgencia/emergencia. El embarazo en la adolescencia es un problema de salud importante. Los hábitos tóxicos, alimentarios y culturales con deficiencia son factores de riesgo en la aparición de enfermedades en la comunidad.

Palabras clave: Medicina Comunitaria, Epidemiología.

\section{ABSTRACT}

Epidemiological study in the Entre Rios Health Area

Objective. To determine the major diseases and health problems in the rural community of Entre Ríos.

Design. Cross-sectional descriptive study.

Setting. Primary care. Health area of Entre Ríos Village, Caroebe, RR, Brazil.

Main measurements. Socio-demographic and morbidity data in patients with a diagnosis of chronic diseases, outpatient and emergency visits and risk factors are recorded.

Results: There was a predominance of cases aged $0-10$ years $(36.09 \%)$ and of female gender $(61.4 \%)$. Patients with chronic diseases identified in the Hypertension and Diabetes Mellitus programme constituted $2.98 \%$ (72 cases). Tropical diseases: malaria, dengue fever, leishmaniasis are frequent with 16 cases $(0.66 \%), 20$ cases $(0.81 \%)$, and 24 cases $(0.99 \%)$ respectively. Other respiratory and intestinal type diseases constitute $13.26 \%$ and $10.36 \%$ respectively. There were 41 pregnant teenagers (15-18 years), $66 \%$ of cases seen in the Prenatal Programme.

Conclusions. Patients classified with non-communicable diseases are controlled and receive consistent healthcare. There is a predominance of patients with communicable diseases, mainly acute respiratory and intestinal infections. Tropical diseases such as malaria, dengue fever, and leishmaniasis are common health problems in this region. Of all patients, $63.28 \%$ received emergency healthcare. Pregnancy in adolescence is an important health problem. Toxic habits, poor nutrition and lack of education are risk factors for the occurrence of diseases in this community.

Keywords: Community Medicine, Epidemiology. 


\section{INTRODUCCIÓN}

El mundo actual sufre cambios constantes y muchas veces radicales. Preservar la salud de los pueblos en este contexto es tarea primordial de la cual se obtienen éxitos parciales. En el Brasil, aunque con resultados favorables en los últimos años, como la continua reducción de la mortalidad infantil y el control de las enfermedades inmunoprevenibles, la deuda social en la salud todavía es enorme, por eso conseguir el control de enfermedades y sus riesgos en la población implica conseguir que el sistema de salud acepte las normativas nacionales para el diagnóstico, prevención y tratamiento de enfermedades, garantizando el beneficio de la población en general. Las políticas públicas pasaron a valorizar la familia y estimular los estudios sobre contextos familiares y estrategias de intervención, considerando los orígenes y las repercusiones del vivir en familia en la determinación del proceso salud-enfermedad ${ }^{1}$.

Hace muchas décadas la literatura describe que la familia es considerada como unidad portadora de necesidades que, si no son satisfechas, pueden desencadenar problemas de salud, y también sirve como un recurso o un apoyo para superarlos o para el tratamiento de las enfermedades².

Con la implantación en 1994 del Programa de Salud de la Familia (PSF) se orientan las estrategias a los servicios de atención básica de la salud. En esta época 29.000 agentes comunitarios eran los responsables de la implantación de un módulo de asistencia que sustituyó a las antiguas prácticas basadas en la valorización del Hospital, viendo la enfermedad como foco, ahora dirigido a la promoción de la salud y la participación de la comunidad ${ }^{1}$.

La asistencia a la salud en los países en desarrollo es afectada por la pobreza, inestabilidad política y las enfermedades que pueden tener menor importancia en países industrializados. Estudios demuestran que en los países en desarrollo predominan las enfermedades infecciosas: casos reportados de pacientes en Phnom Penh, en Camboya, con histoplasmosis, meningitis criptocócica, sarna, lesiones cerebrales y virus del SIDA, y de pacientes en Kabul, en Afganistán, con cirrosis hepática y síndrome nefrítico ${ }^{3}$.

En los Estados Unidos, en la década de los años 70, la Academia Americana de Médicos de Familia, considera la atención Primaria de la Salud como "el primer contacto y atención integral y continua en la relación médico-paciente"4.
En la estrategia de salud de la familia el Programa de Salud de la Familia en Brasil constituye el centro principal en el contacto directo con la familia y la comunidad, permitiendo conocer en primer plano el desarrollo de las enfermedades, su identificación, los riesgos y la prevención, lo que permite el diagnóstico de la situación de salud en la localidad, enfatizando en los programas de las enfermedades transmisibles (DST/VIH SIDA, Dengue, Malaria, Leishmaniosis), y programas de enfermedades no transmisibles y factores de riesgo (hipertensión arterial, diabetes mellitus, atención Integral a niños, ancianos, embarazadas).

En Roraima, Estado brasileño localizado en la región norte del país que integra la Amazonia Occidental, predominan enfermedades como: malaria, dengue, hepatitis, enfermedad de Chagas, oncocercosis, tuberculosis y enfermedad de Hansen. El SIDA tiene presencia importante entre las enfermedades. Otras enfermedades, como sarampión, tétanos, varicela, rubeola, meningitis y leishmaniosis también surgen con frecuencia ${ }^{5}$.

Nuestro trabajo en el Programa de Salud de la Familia (PSF) es realizado en el Distrito de Entre Ríos, a $35 \mathrm{~km}$ de distancia del municipio de Caroebe, en la Región sur del Estado de Roraima, en las cercanías de la selva Amazónica. El mismo tiene un área de $12.098,5 \mathrm{~km}^{2}$ y dista de la capital del Estado 354 $\mathrm{km}$; su economía es fundamentalmente agropecuaria, su población es de 5.901 habitantes y posee un clima tropical lluvioso y con estación seca. El acceso a la Villa de Entre Ríos desde la Sede municipal es realizado por la carretera sin asfalto BR 210, y comprende diferentes comunidades con una población de aproximadamente 2.000 habitantes.

Basados en parámetros geográficos, demográficos, culturales y sociales, la comunidad solo es consciente de sus problemas de salud cuando es capaz de identificarlos, procurando priorizar y desarrollar planes de acción para solucionarlos y/o prevenirlos. Por eso, consideramos necesario realizar este trabajo para conocer las principales enfermedades y problemas de salud en la comunidad rural de Entre Ríos. En nuestra labor cotidiana en los servicios directos con la comunidad nos percatamos de dichos problemas de salud y así desarrollamos estrategias sanitarias para fortalecer la Atención Primaria de Salud.

\section{MATERIAL Y MÉTODO}

El presente trabajo es de carácter descriptivo, utilizando el análisis cuantitativo de los datos obtenidos 
de las historias clínicas, fichas de control y visitas domiciliarias.

El estudio fue realizado en el Centro de Salud del Distrito de Entre Ríos, municipio de Caroebe, Estado de Roraima (Brasil), que desarrolla actividades de salud de tipo ambulatorio y servicios de urgencia con atendimientos de hasta complejidad media. Es una entidad municipal mantenida por financiamiento de la alcaldía municipal y recursos de los Programas de Estrategia de Salud de la Familia del Ministerio de Salud y el Gobierno Federal. Posee e incluye servicios de vacunación, sala de urgencia/ emergencia, de farmacia, de consultas médicas, de enfermería, de estomatología, laboratorio de microscopía y para colección de muestras de exámenes que son realizados en el Laboratorio del Hospital de Caroebe, ciudad sede del municipio.

Los sujetos de estudio fueron los pacientes controlados en los programas de hipertensión arterial y diabetes mellitus y pacientes atendidos de forma ambulatoria en consultas médicas y de enferme- ría en el periodo de enero-diciembre de 2009, así como pacientes atendidos en el servicio de urgencia/emergencia.

En cuanto a los procedimientos metodológicos, los datos fueron obtenidos mediante un cuestionario y posteriormente introducidos en una base de datos, utilizando el programa Epi-info 2007. El análisis de los datos fue realizado a través del programa Excel 2007. Para analizar y agrupar las alteraciones de salud identificadas fue utilizada la Clasificación Internacional de Enfermedades y Problemas relacionados con la Salud (ICD-10) de 1993. La distribución de frecuencias de las variables investigadas fue expresada en porcentaje.

\section{RESULTADOS}

En la tabla 1 se observa que predominaron los pacientes atendidos del grupo etario de 0-10 años de edad $(36,09 \%)$ y del sexo femenino ( $61,4 \%)$, siguiendo en frecuencia los jóvenes y adultos mayores $(19,47 \%)$ respectivamente.

\begin{tabular}{l|c|c|c}
\hline \multirow{2}{*}{ Grupos etarios } & \multicolumn{2}{|c|}{ Sexo } & \multirow{2}{*}{ Total } \\
\cline { 2 - 3 } & Masculino & Femenino & \\
\cline { 2 - 3 } & $\mathbf{N}^{\circ}(\%)$ & $\mathbf{N}^{\circ}(\%)$ & $\mathbf{N}^{\circ}(\%)$ \\
\hline \multirow{2}{*}{$0-10$} & $281(11,6)$ & $590(24,5)$ & $871(36,1)$ \\
$11-20$ & $190(7,9)$ & $250(10,4)$ & $440(18,2)$ \\
$21-40$ & $185(7,7)$ & $285(11, .8)$ & $470(19,5)$ \\
$41-50$ & $182(7,5)$ & $288(11,9)$ & $470(19,5)$ \\
$51-60$ & $62(2,6)$ & $40(1,7)$ & $102(4,2)$ \\
60 y más & $30(1,2)$ & $30(1,2)$ & $60(2,5)$ \\
\hline Total & $930(38,5)$ & $1483(61,4)$ & $2413(100,0)$ \\
\hline
\end{tabular}

Tabla 1. Distribución de los pacientes según edad y sexo.

\begin{tabular}{l|c}
\hline Enfermedades & $\mathbf{N}^{\circ}$ de pacientes (\%) \\
\hline Hipertensión arterial & $49(2,0)$ \\
Diabetes mellitus I & $3(0,1)$ \\
Diabetes mellitus II & $20(0,8)$ \\
\hline Total & $72(3,0)$ \\
\hline
\end{tabular}

Tabla 2. Distribución de los pacientes según clasificación de enfermedades crónicas no transmisibles. 


\begin{tabular}{l|c}
\hline Enfermedades & $\boldsymbol{N}^{\mathbf{0}}$ de pacientes (\%) \\
\hline Infecciones respiratorias agudas (no tuberculosas) & $320(13,3)$ \\
Infecciones intestinales & $250(10,4)$ \\
Leishmaniosis & $24(1,0)$ \\
Dengue & $20(0,8)$ \\
Enfermedades sexualmente transmisibles (ETS) & $18(0,7)$ \\
Paludismo (malaria) & $16(0,7)$ \\
Hepatitis virales & $05(0,2)$ \\
Tuberculosis & $0(0)$ \\
\hline Total & $635(26.3)$ \\
\hline
\end{tabular}

Tabla 3. Distribución de los pacientes según clasificación de enfermedades transmisibles.

\begin{tabular}{l|c}
\hline Atendimiento & $\boldsymbol{N}^{\mathbf{0}}$ de pacientes (\%) \\
\hline Urgencia/Emergencia & $1527(63,3)$ \\
Ambulatorio & $861(35,7)$ \\
Remitidos al hospital & $25(1,0)$ \\
\hline Total & $2413(100,0)$ \\
\hline
\end{tabular}

Tabla 4. Distribución de pacientes según tipo de atendimiento.

\begin{tabular}{l|c}
\hline Enfermedades & $N^{\circ}$ de pacientes (\%) \\
\hline Enfermedades cardiovasculares no hipertensivas. & $71(2.9)$ \\
Traumas & $25(1.0)$ \\
Accidentes por animales pesoñentos & $15(0.6)$ \\
Enfermedades respiratorias crónicas & $05(0,2)$ \\
Enfermedad de Hansen & $02(0.1)$ \\
Otras (hepatopatias no virales, hematopatías, & $230(9.5)$ \\
artropatías, nefropatías, dermatitis agudas) & \\
\hline Total & $348(14.4)$ \\
\hline
\end{tabular}

Tabla 5. Clasificación atendiendo a otros tipos de enfermedades diagnosticadas en el ambulatorio.

En la tabla 2 observamos que de las enfermedades crónicas no transmisibles la hipertensión arterial supuso un $2,03 \%$ de los pacientes, la diabetes tipo 1 un $0,12 \%$ y la diabetes tipo 2 un $0,82 \%$, constituyendo un total de 72 casos $(2,98 \%)$ del universo de pacientes con atendimiento ambulatorio.

El comportamiento de las enfermedades transmisibles identificadas y que constituyeron problemas de salud en esta comunidad se presenta en la tabla 3 , donde predominan las infecciones respiratorias no tuberculosas (320 pacientes) y las infecciones intestinales (250 pacientes). Predominaron las enfermedades tropicales, como malaria $(0,66 \%)$, dengue $(0,82 \%)$ y leishmaniosis $(0,99 \%)$, al existir en esta región típica del Amazonas una elevada frecuencia de pacientes afectados. Las enfermedades sexualmente transmisibles y las hepatitis virales constituyeron el $0,74 \%$ y el $0,20 \%$ respectivamente. 


\begin{tabular}{l|c}
\hline Grupos de edades & No de embarazadas (\%) \\
\hline $12-14$ & $02(8.3)$ \\
$15-18$ & $10(41.7)$ \\
$19-25$ & $05(20.8)$ \\
$26-30$ & $05(20.8)$ \\
31 y más & $02(8.3)$ \\
\hline Total & $24(100,0)$ \\
\hline
\end{tabular}

Tabla 6. Identificación de las pacientes embarazadas según la edad.

\begin{tabular}{l|c}
\multicolumn{1}{c|}{ Factores de riesgo } & $\boldsymbol{N}^{\circ}$ de pacientes (\%) \\
\hline Hábitos nutricionales (alimentación insuficiente) & $1676(69.5)$ \\
Nivel de escolaridad: & $1200(49.7)$ \\
- Primario & $350(14.5)$ \\
- Medio & $40(1.7)$ \\
- Superior & $140(5.8)$ \\
Información - cultura. & $350(14.5)$ \\
Hábitos tóxicos: & $10(0.4)$ \\
- Bebidas alcohólicas & $\mathbf{2 4 1 3}$ \\
\hline \multicolumn{1}{c}{ - Tabaquismo. } & $\mathbf{2 1 3}$ \\
\hline
\end{tabular}

Tabla 7. Identificación de los factores de riesgo.

En la tabla 4 se distribuyen los pacientes con atendimiento de urgencia/emergencia $(63,28 \%)$, ambulatorio (35,68\%) y traslado al Hospital base $(1,04 \%)$, lo que demuestra el aprovechamiento, todavía en difíciles condiciones de infraestructura y recursos sanitarios, del personal de salud en este local, pues debido a la distancia (35 km con camino sin asfalto y malas condiciones) es preciso hacer servicios ambulatorios (consultas médicas) interrumpidos muchas veces para ofrecer atendimiento de urgencia/emergencia en cualquier horario y en cualquier momento.

En la tabla 5 identificamos otras enfermedades que fueron también motivo de consulta, donde predominan las afecciones cardiovasculares no hipertensivas, como insuficiencia cardiaca $(2,94 \%)$, así como las afecciones respiratorias crónicas EPOC y bronquiectasias $(0,20 \%)$, picaduras por animales pesoñentos (cobras, escorpiones) $(0,62 \%)$, atendimientos de emergencia por traumas por accidentes de tránsito y otros $(1,03 \%)$, así como otras enfer- medades con menos prevalencia, como encefalopatías, hepatopatías no virales, dermatopatias agudas y nefropatías $(9,53 \%)$.

En la tabla 6 mostramos el número de gestantes atendidas según el programa de Prenatal, donde predominaron los casos de embarazo en la adolescencia: 10 casos en el grupo de edad 15-18 años $(41,68 \%)$.

Identificamos los factores de riesgo que muestra la tabla 7, los cuales inciden directa o indirectamente en los problemas de salud diagnosticados, incluyendo la ingestión de bebidas alcohólicas (14,50\%), hábitos alimentares insuficientes $(69,45 \%)$ y nivel de escolaridad con enseñanza primaria concluida o no $(49,73 \%)$.

\section{DISCUSIÓN}

Un estudio de pacientes atendidos en la Universidad Federal de Santa Catarina ${ }^{6}$ demostró el predominio de familias cuyos miembros poseían entre 
31-40 años, siguiendo en frecuencia los grupos de edad de 41-60 años, lo que caracteriza una clientela todavía joven y que, en cierto modo, reproduce la pirámide demográfica del país, particularmente de la Región Sur del Brasil.

La literatura plantea que las enfermedades cardiovasculares tienen relación directa y proporcional con la edad. La hipertensión arterial es la enfermedad más frecuente, predominando la hipertensión sistodiastólica y la hipertensión sistólica aisladas en personas de la tercera edad, y con prevalencia de hasta un $15 \%{ }^{7,8}$.

La diabetes mellitus tiene elevada prevalencia mundial y la estimación actual del número de pacientes con esta enfermedad en el mundo es de 170 millones. La estimación en Brasil es que existen entre 7 y 8 millones de pacientes, de los cuales el $90 \%$ son del tipo 2 , el $8-9 \%$ del tipo 1 y el restante $2 \%$ comprende las formas raras o secundarias y/o asociadas a otros síndromes ${ }^{8,9}$.

Las infecciones respiratorias agudas representan una de las primeras causas de atención médica a nivel mundial. Estadísticas disponibles indican que entre $30-60 \%$ de las consultas de niños enfermos son motivadas por este tipo de infección, así como en las personas mayores de 60 años $^{10}$. La OMS y la Oficina Regional para las Américas definieron un programa de control de las infecciones respiratorias agudas, en el cual establecen diferentes objetivos con la estrategia siguiente: disminuir el uso de antibióticos en menores de 5 años, reducir la frecuencia de sus complicaciones y reducir su incidencia y su gravedad. La OMS recomienda la vacunación contra la influenza para personas en riesgo, medida efectiva para reducir la enfermedad ${ }^{11}$.

En el Brasil el comportamiento de las enfermedades respiratorias agudas e intestinales, según estudios realizados, continúa siendo un grave problema de salud, donde los Programas de Salud de la Familia (PSF), a través de la práctica de la medicina preventiva en las diferentes comunidades rurales y urbanas, permitirán su reducción paulatina ${ }^{12,13}$.

Los casos sospechosos y/o confirmados de dengue son notificados a la Vigilancia Epidemiológica, pues esa enfermedad es dinámica, siendo adaptado el protocolo de conductas en el atendimiento e identificación precoz de los $\operatorname{casos}^{14}$ e implementándose medidas oportunas para el control de la enfermedad. Un estudio realizado en Rio de Janeiro muestra la coincidencia de picos de temperatura mínima con el inicio de las epidemias de dengue en el periodo de 1986 a $2003^{15}$. Otro estudio refleja el aumento de la incidencia de casos de dengue confirmados en los periodos de lluvias, de enero a julio, en Manaos, estado de Amazonas, donde fueron analizados 482 casos $^{16}$.

La leishmaniosis constituye una enfermedad típica tropical y frecuente en zonas rurales de la selva amazónica. Al revisar la literatura se encontró la ocurrencia de 3 brotes en las regiones este y sudeste del estado de Sao Paulo en los años 1993-1995 y 2002-2003, con perfil semejante, transmisión peridomiciliar, proximidad del domicilio al campo y tiempo de convivencia. La Leishmaniosis Tegumentaria Americana (LTA) presenta coeficientes de detección con tendencia al crecimiento, que oscilaron entre 3,8 y 22,9 para 100.000 habitantes $^{17}$.

Las hepatitis de etiología viral son uno de los mayores problemas de salud pública en todo el mundo. La infección por el virus de la hepatitis $B$ es endémica en muchas partes del mundo, estimándose que existen 300 millones de portadores crónicos de este virus, cerca de un $5 \%$ de la población mundial| ${ }^{18,19}$.

En la actualidad, la incidencia de las Enfermedades de Transmisión Sexual (ETS) viene aumentando. En los estados Unidos, algunos autores refieren que la prevalencia de ETS entre adolescentes debe ser en torno al $25 \%$ y el grupo etario de 15-24 años es el de mayor riesgo ${ }^{3}$. Los equipos de atención básica tienen un papel relevante en el diagnóstico y en el acompañamiento de las personas con estas enfermedades, identificando los casos sospechosos y contactos, solicitando exámenes de laboratorio adecuados y realizando interconsultas a servicios de referencia en los casos indicados, así como la promoción a la salud y prevención.

La estrategia de salud de la familia implantada en el Brasil permite la atención ambulatoria independientemente de la atención de urgencia/emergencia que tiene lugar en el hospital o Prontos Socorros, tanto en regiones urbanas como rurales, además de la aplicación de los diferentes programas del Ministerio de Salud: malaria o paludismo, hipertensión, diabetes y prenatal. Este último es de suma importancia pues la gravidez en la adolescencia es un problema de salud complejo, aumentando todavía más en las comunidades rurales que en las grandes metrópolis, motivado entre otras causas por las dificultades socio económicas, falta de calidad en la educación en general, así como los factores socio culturales que aceptan esta condición en la mujer como rasgo característico del ambiente familiar ${ }^{1,3}$. 
A través de las visitas domiciliares se permite hacer un abordaje familiar, superando la práctica centrada exclusivamente en la enfermedad, visualizando la familia como recurso y unidad promotora de cuidado ${ }^{12}$.

En un estudio realizado en el estado de Santa Catarina se identifican los riesgos intra-familiares de 322 pacientes atendidos en el ambulatorio de medicina familiar y comunidad de la UNIVALI, UFSC ${ }^{3}$, en el periodo 2003-2005, destacando: edad, baja o ninguna escolaridad, grupos vulnerables (niños y ancianos), tabaquismo, alcoholismo, uso de drogas, riesgos psicosociales, estructuración familiar y riesgos genéticos, morbilidad y comorbilidad, enfermedades crónicas (diabetes e hipertensión arterial), permitiendo comprender el contexto familiar y verificando los patrones de repetición de hábitos de vida, relaciones intrafamiliares y patologías hereditarias que interfieren en el proceso salud-enfermedad, adaptando estrategias a los riesgos identificados.

La baja escolaridad es considerado un gran factor de riesgo en la familia, pues disminuye su repertorio de búsqueda de información para el enfrentamiento de los problemas que surjan a lo largo del ciclo vital, limitando las posibilidades terapéuticas y de cuidado a la familia ${ }^{1}$, y encontrándose una relación directa entre baja escolaridad, baja renta y mayor vulnerabilidad a las enfermedades. Otros estudios demuestran que el tabaquismo predomina en el $32,9 \%$ de las familias estudiadas y el uso de alcohol y drogas en el $26,1 \%{ }^{4}$.

Una alimentación saludable es primordial en la aparición o no de enfermedades. El consumo de sal es considerado abusivo y peligroso en Brasi $\left.\right|^{3,5}$, donde el consumo per cápita llega a $12 \mathrm{gr}$ diarios y según la OMS el consumo individual no debe exceder a $2 \mathrm{gr}$ diarios. Entre las consecuencias del consumo excesivo de sal se encuentran: retención de líquido, aumento del volumen de sangre en las arterias y elevación de la presión arterial.

Como conclusiones, predominaron los pacientes atendidos menores de 10 años y del sexo femenino, el $2,98 \%$ de los pacientes atendidos están clasificados con enfermedades crónicas no transmisibles, el 23,3\% fueron atendidos por enfermedades transmisibles, encontrándose las infecciones respiratorias e intestinales agudas como predominantes. Las enfermedades tropicales como paludismo, dengue y leishmaniosis constituyen problemas de salud típicos en esta región. Del total de pacientes, el $63,28 \%$ recibió atención de urgencia/emergencia. El embarazo en la adolescencia predomina, siendo un problema de salud importante en la comunidad estudiada. Por último, los hábitos tóxicos, alimentarios y culturales con deficiencia son factores de riesgo en la aparición de enfermedades en la comunidad.

\section{BIBLIOGRAFÍA}

1. Duncan BB, Schmidt MI, Giugliani ERJ. Medicina ambulatorial: conduta clínica em atenção primaria. $3^{a}$ ed. Porto Alegre: Artes Acadêmicas; 2004.

2. Rodríguez A. Familia y Comunidad. Rev Cuba Med Gen Integr. 2007; 23(3):30-5.

3. Rouquayrol Z, Almeida FN. Epidemiologia é Saúde. $6^{a}$ ed. Rio de Janeiro: Medsi; 2003.

4. Jardines J, Padrón L. La especialidad de Medicina General Integral. Rev Cuba Med Gen Integr. 1991; 7(2):108-14.

5. Conselho Regional de Medicina. Estadísticas. Disponible en: http://www.portalmedico.com.br. [Acceso el 23 de noviembre de 2009].

6. Lessa I. Epidemiologia da hipertensão. In: Lessa I. O adulto brasileiro e as doenças da modernidade. Epidemiologia das doenças crônicas não transmissíveis. São Paulo, Rio de Janeiro: Hucitec, Abrasco; 1998. p. 77-96.

7. Reyes P. Morbilidad cardiovascular en el adulto mayor. Rev Cuba Med Gen Integr. 2001; 17(7):21- 6.

8. Ministério da Saúde. Brasil. Departamento de ações programáticas estratégicas. Plano de reorganização da atenção básica a hipertensão arterial e ao diabetes mellitus: Manual da HÁ e DM. Brasília (DF): Ministério da Saúde; 2001.

9. White Jr. Therapy for Diabetes Mellitus. American Diabetes Association - Clinical Education Series - Fourth Edition; 2007.

10. Murahovschi, J. Pediatria, Diagnostico e Tratamento. $6^{a}$ ed. São Paulo: Ed. Sarvier; 2003.

11. Ministério da Saúde. Brasil. Doenças infecciosas e parasitarias. Guia de bolso. Brasília: Ministério da Saúde; 2006.

12. Carta de Otawa para a Promoção da Saúde. I Conferência Internacional sobre Promoção da Saúde, 1986. Disponible em: http://www.opas.org.br/promocao/uploadArq/Ottawa. pdf

13. Cueto GA, Pérez MC, Mildestein S, Núñez ME, Alegret M. Martínez MR. Características del parasitismo intestinal en niños de las comunidades del policlínico XX Aniversario. Rev Cuba Med Gen Integr. 2009; 25(1). Disponible en: http://scielo.sld.cu/pdf/mgi/v25n1/mgi08109.pdf

14. Gubler DJ, Kuno G. Dengue and hemorrhagic fever. Ist Ed. New York: CABI Publishing; 2001.

15. Portela F, Fagundes A, Teixeira G, Cardoso D. Clima e epidemias de dengue no estado de Rio de Janeiro. Ver Soc Bras Med Trop. 2009; 42(2). Disponible em: http://www. scielo.br/pdf/rsbmt/v42n2/v42n2a08.pdf.

16. Rocha LA, Tauil P. Dengue em Criança: aspectos clínicos e epidemiológicos, Manaus, Estado de Amazonas, no período de 2006-2007. Ver Soc Bras Med Trop. 2009; 42(1). Disponible em: http://www.scielo.br/pdf/rsbmt/v42n1/ v42n1a05.pdf.

17. Augusto $L$ et al. Vigilância epidemiológica de doenças tropicais. Ver Bras De Saúde. 1996; 14(54):32-64.

18. Ministério da Saúde. Brasil. Hepatites virais: O Brasil esta atento. Brasília: Ministério da Saúde; 2008.

19. Melo L, Ferraz JC, Bothelo R, Silva W, Madeiros L. Prevalência de marcadores para o vírus da hepatites $B$ em contatos domiciliares no Estado de Amazonas. Ver Soc Bras Med Trop. 2003; 36(5). http://www.scielo.br/pdf/rsbmt/ v36n5/a04v36n5.pdf. 